\title{
Solving dual flexible job-shop scheduling problem using a Bat Algorithm
}

\author{
Xu, H. ${ }^{\text {a, }}{ }^{*}$, Bao, Z.R. ${ }^{a}$, Zhang, T. ${ }^{a}$ \\ ${ }^{a}$ School of Internet of Things Engineering, Jiangnan University, Wuxi, China
}

\section{A B S T R A C T}

For the flexible job-shop scheduling problem with machine selection flexibility and process sequence flexibility in process design, types and characteristic of machine selection and process sequence flexibility are analyzed. The mathematical model of dual flexible job-shop scheduling problem is established, and an improved bat algorithm is proposed. For purpose of expressing the relationship effectively between the process and the bat population, a new method of encoding strategy based on dual flexibility degree is proposed. The crossover and mutation operation are designed to strengthen the searching ability of the algorithm. For purpose of overcoming the shortcomings of the fixed parameters in bat algorithm, the value of the inertia weight was adjusted, and a linear decreasing inertia weight strategy was proposed. We carried out experiments on actual examples, it can be seen from the experimental results that the robustness and optimization ability of the algorithm we proposed are better than Genetic Algorithm (GA) and Discrete Particle Swarm Optimization algorithm (DPSO). This shows that the proposed algorithm is more excellent in solving the flexible job-shop scheduling problem, and it is an efficient scheduling algorithm.
\end{abstract}

\section{ARTICLE INFO}

Keywords:

Flexible job-shop scheduling

Optimization

Process sequence flexibility

Machine selection flexibility

Bat algorithm

Genetic algorithm

Particle swarm optimization

*Corresponding author:

joanxh2003@163.com

(Xu, H.)

Article history:

Received 16 November 2016

Revised 13 February 2017

Accepted 15 February 2017

(C) 2017 PEI, University of Maribor. All rights reserved.

\section{References}

[1] Röck, H. (1984). The three-machine no-wait flow shop problem is NP-complete, Journal of the ACM, Vol. 31, No. 2, 336-345, doi: 10.1145/62.65.

[2] Yuan, Y., Xu, H., Yang, J. (2013). A hybrid harmony search algorithm for the flexible job shop scheduling problem, Applied Soft Computing, Vol. 13, No. 7, 3259-3272, doi: 10/1016/j.asoc.2013.02.013.

[3] Zhao, S.-K., Fang, S.-L., Gu, X.-J. (2013). Genetic algorithm with new initialization mechanism for flexible job shop scheduling, Journal of Zhejiang University (Engineering Science), Vol. 47, No. 6, 1022-1030, doi: 10.3785/i.issn. 1008-973X.2013.06.013.

[4] Zhao, S.-K., Fang, S.-L., Gu, X.-J. (2014). Machine selection and FJSP solution based on limit scheduling completion time minimization, Computer Integrated Manufacturing Systems, Vol. 20, No. 4, 854-865, doi: 10.13196/i.cims. 2014.04.zhaoshikui.0854.12.20140416.

[5] Luan, F., Wang, W., Fu, W.P., Bao, Y.T., Ren, G.C., Wang, J., Deng, M.M. (2014). FJSP solving by improved GA based on PST hierarchy structure, Computer Integrated Manufacturing Systems, Vol. 20, No. 10, 2494-2501.

[6] Saygin, C., Kilic, S.E. (1999). Integrating flexible process plans with scheduling in flexible manufacturing systems, The International Journal of Advanced Manufacturing Technology, Vol. 15, No. 4, 268-280, doi: 10.1007/ s001700050066.

[7] Huang, X.W., Zhao, X.Y., Ma, X.L. (2014). An improved genetic algorithm for job-shop scheduling problem with process sequence flexibility, International Journal of Simulation Modelling, Vol. 13, No. 4, 510-522, doi: $10.2507 /$ ijsimm13(4)co20.

[8] Ba, L., Li, Y., Yang, M.S., Gao, X.Q., Liu, Y. (2016). Modelling and simulation of a multi-resource flexible job-shop scheduling, International Journal of Simulation Modelling, Vol. 15, No. 1, 157-169, doi: 10.2507/IJSIMM15(1)CO3. 
[9] Modrák, V. Pandian, R.S. (2010). Flow shop scheduling algorithm to minimize completion time for n-jobs mmachines problem, Tehnički vjesnik -Technical Gazette, Vol. 17, No. 3, 273-278.

[10] Yang, X.-S. (2010). A new materialistic bat-inspired algorithm, Nature Inspired Cooperative Strategies for Optimization, Vol. 284, 65-74, doi: 10.1007/978-3-642-12538-6 6.

[11] Ali, E.S. (2014). Optimization of power system stabilizers using bat search algorithm, International Journal of Electrical Power \& Energy Systems, Vol. 61, 683-690, doi: 10.1016/j.ijepes.2014.04.007.

[12] Shi, H., Wang, W., Li, Y., Lu, L. (2015). Bat algorithm based on Lévy flight feature and its localization application in WSN, Chinese Journal of Sensors and Actuators, Vol. 6, 888-894, doi: 10.3969/j.issn.1004-1699.2015.06.019.

[13] Chen, Y., Wang, Z., Wang, Z. (2014). Feature selection of infrared spectrum based on improved bat algorithm, Infrared and Laser Engineering, Vol. 43, No. 8, 2715-2721, doi: 10.3969/i.issn.1007-2276.2014.08.054.

[14] Chen, Y., Wang, Z., Wang, Z. (2015). Mind evolutionary bat algorithm and its application to feature selection of mixed gases infrared spectrum, Infrared and Laser Engineering, Vol. 3, 845-851, doi: 10.3969/j.issn.1007-2276. 2015.03.010.

[15] Yang, X.-S. (2011). Bat algorithm for multi-objective optimization, International Journal of Bio-Inspired Computation, Vol. 3, No. 5, 267-274, doi: 10.1504/IJBIC.2011.042259.

[16] Komarasamy, G., Wahi, A. (2012). An optimized k-means clustering technique using bat algorithm, European Journal of Scientific Research, Vol. 84, No. 2, 263-273.

[17] Yang, X.-S., Gandomi, A.H. (2012). Bat algorithm: A novel approach for global engineering optimization, Engineering Compotations, Vol. 29, No. 5, 464-483, doi: 10.1108/02644401211235834.

[18] Marichelvam, M.K., Prabaharan, T. (2012). A bat algorithm for realistic hybrid flowshop scheduling problems to minimize makespan and mean flow time, ICTACT Journal on Soft Computing, Vol. 3, No. 1, 428-433, doi: 10.21917 Цijsc.2012.0066.

[19] Marichelvam, M.K., Prabaharan, T., Yang, X.-S., Geetha, M. (2013). Solving hybrid flow shop scheduling problems using bat algorithm, International Journal of Logistics Economics and Globalization, Vol. 5, No. 1, 15-29, doi: 10.1504 /IJLEG.2013.054428.

[20] Luo, Q., Zhou, Y., Xie, J., Ma, M., Li, L. (2014). Discrete bat algorithm for optimal problem of permutation flow shop scheduling, The Scientific World Journal, Vol. 2014, 1-15, doi: 10.1155/2014/630280.

[21] Zhang, J.J., Li, Y.G. (2014). An improved bat algorithm and its application in permutation flow shop scheduling problem, Advanced Materials Research, Vol. 1049-1050, 1359-1362, doi: 10.4028/www.scientific.net/amr.10491050.1359.

[22] Dekhici, L., Belkadi, K. (2015). A bat algorithm with generalized walk for the two-stage hybrid flow shop problem, International Journal of Decision Support System Technology, Vol. 7, No. 3, 1-16, doi: 10.4018/ijdsst.2015070101.

[23] Mirjalili, S., Mirjalili, S.M., Yang, X.-S. (2014). Binary bat algorithm, Neural Computing and Applications, Vol. 25, No. 3, 663-681, doi: 10.1007/s00521-013-1525-5.

[24] Bai, J., Gong, Y.-G., Wang, N.-S., Tang, D.-B. (2010). Multi-objective flexible job shop scheduling with lot-splitting, Computer Integrated Manufacturing Systems, Vol. 16, No. 2, 396-403.

[25] Zhao, S. (2015). Bilevel neighborhood search hybrid algorithm for the flexible job shop scheduling problem, Journal of Mechanical Engineering, Vol. 51, No. 14, 175-184.

[26] Xu, H., Zhang, T. (2015). Improved discrete particle swarm algorithm for solving flexible flow shop scheduling problem, Journal of Computer Application, Vol. 35, No. 5, 1342-1347, doi: 10.11772/j.issn.1001-9081.2015.05. 1342.

[27] Cui, J.S., Li, T.K., Zhang, W.X. (2005). Hybrid flow shop scheduling model and its genetic algorithm, Journal of University of Science and Technology Beijing, Vol. 27, No. 5, 623-626, doi: 10.3321/i.issn:1001-053X.2005.05.027. 


\section{APEM}

Advances in Production Engineering \& Management

Letnik 12 | Številka 1 | Marec 2017 | Strani 5-16

https://doi.org/10.14743/apem2017.1.235
ISSN 1854-6250

Spletna stran: apem-journal.org Izvirni znanstveni članek

\title{
Reševanje dvojno prilagodljivega problema načrtovanja proizvodnje $z$ uporabo algoritma po vzoru obnašanja netopirjev
}

\author{
Xu, H. ${ }^{\mathrm{a},{ }^{*}}$, Bao, Z.R. ${ }^{\mathrm{a}}$, Zhang, T. $^{\mathrm{a}}$ \\ ${ }^{\mathrm{a}}$ School of Internet of Things Engineering, Jiangnan University, Wuxi, China
}

\section{POVZETEK}

Raziskani so tipi in značilnosti izbire strojev in prilagodljivosti zaporedja procesov, za problem prilagodljivega načrtovanja proizvodnje s prilagodljivo izbiro strojev in s prilagodljivim zaporedjem procesov $v$ fazi načrtovanja procesa. Predstavljen je matematični model dvojno prilagodljivega načrtovanja proizvodnje, prav tako pa nadgrajen netopirski algoritem. Za opis učinkovitosti razmerja med procesom in populacijo netopirjev, je vpeljana nova metoda strategije kodiranja, ki temelji na stopnji dvojne prilagodljivosti. Za okrepitev iskalne sposobnosti algoritma sta vključeni operaciji križanja in mutacije. Ker konstantne vrednosti spremenljivk predstavljajo slabost netopirskega algoritma, je vrednost vztrajnostne teže prilagojena, tako da se linearno zmanjšuje. Poskusi na realnih primerih kažejo, da je predlagan algoritem bolj robusten in ima boljšo optimizacijsko sposobnost kot genetski algoritem (GA) ali diskretni algoritem z rojem delcev (angl. DPSO). To dokazuje, da je predlagan algoritem primernejši za reševanje prilagodljivega načrtovanja proizvodnje in je učinkovit pri načrtovanju.

(C) 2017 PEI, University of Maribor. All rights reserved.

\section{PODATKI O ČLANKU}

Ključne besede:

Prilagodljivo načrtovanje proizvodnje

Optimizacija

Prilagodljivo zaporedje procesov

Prilagodljiva izbira strojev

Netopirski algoritem

Genetski algoritem

Algoritem z rojem delcev

* Kontaktna oseba: joanxh2003@163.com

(Xu, H.)

Zgodovina članka:

Prejet 16. novembra 2016

Popravljen 13. februarja 2017

Sprejet 15. februarja 2017 